\title{
CURES OF COUPLED BUNCH INSTABILITIES
}

\author{
A. Mosnier, SOLEIL, Gif/Yvette (France)
}

\section{Abstract}

To achieve the desired luminosities and brilliances in future storage rings for colliders and synchrotron light sources, very large beam currents need to be stored in many tightly spaced bunches. A serious limitation of the performances comes then from the conventional coupled bunch instabilities (CBI). Energy oscillations and transverse ocillations are mainly driven by high-Q structures and the resistivity of the vacuum chamber. Control of these multibunch instabilities has become a critical issue and various strategies are considered to push up the instability thresholds: HOM frequency control to avoid the overlap of dangerous modes with beam spectrum, heavy mode damping to reduce the resonant buildup of the fields, Landau damping to destroy the coherence of the beam and lastly strong feedback systems operating either in the frequency or time domains. These different cures and their technological developments are reviewed in this short paper, more details can be found in [1].

\section{INTRODUCTION}

The common approach of both synchrotron light sources and $\mathrm{e}^{+} \mathrm{e}^{-}$circular colliders to achieve the desired luminosities and brilliances consists in storing high beam currents in many tightly spaced bunches. Control of coupled-bunch oscillations, excited by the long-range parasitic modes of the vacuum chamber, mainly of the $r f$ cavities, has become accordingly a critical issue. Energy or position oscillations spoil the luminosity in colliders, because beams don't collide properly any more, in time or in position, and the spectral brilliance in synchrotron light sources, because undulators are strongly sensitive to any increase in effective energy spread and emittance. Figure 1 shows for example the effect of longitudinal CBI on the brightness of a typical undulator in the SOLEIL light source for different energy oscillation amplitudes.

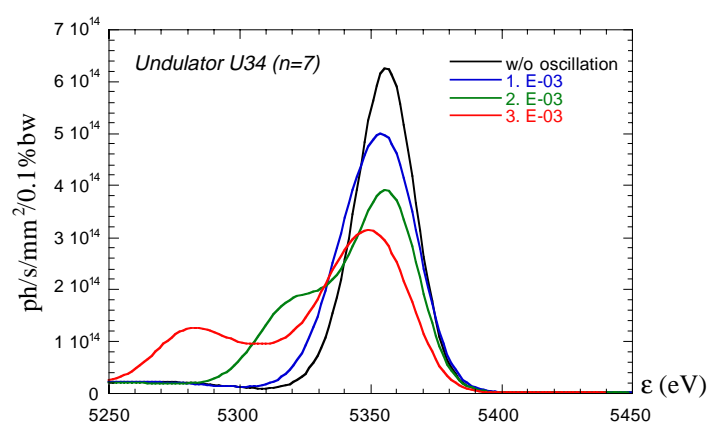

Figure 1: Effect of longitudinal oscillation on the 7th harmonic of SOLEIL U34 undulator spectrum.
When we are interested in the coherent motion of a bunch as a whole, the one-particle model, also named rigid bunch approximation, leads to a well-known system of linear equations (see [2,3,4] for example). For a beam interacting with its environment, the single particle equation of longitudinal motion is given by:

$$
\ddot{\tau}_{k}(t)+2 \alpha_{s} \dot{\tau}_{k}(t)+\omega_{s k}^{2} \tau_{k}(t)=\frac{\alpha}{T_{0} E / e} V_{k}(t)
$$

where $\tau_{k}$ and $\omega_{s k}$ are the time displacement with respect to the synchronous particle and the synchrotron frequency of particle index $k ; T_{0}, E$ and $\alpha$ are the revolution period, the beam energy and the momentum compaction; $\alpha_{s}=1 / \tau_{s}$ is the synchrotron damping rate; $V_{k}$ is the total induced voltage by all circulating particles, seen by bunch $k$.

For $\mathrm{M}$ equally spaced and equally populated rigid bunches, a coherent oscillation of the $k$-th bunch is described by:

$$
\tau_{k}(t)=\hat{\tau} e^{j(\Omega t+k \Delta \phi)}
$$

Signals add up coherently and provide synchrotron sidebands if the bunch-to-bunch phase shift satisfies $\Delta \phi=2 \pi n / M$, where $n$ is called the coupled-bunch mode number. The total induced voltage is then obtained by summing the currents of the $M$ individual bunches of current $I_{b}$ :

$$
V_{k}(t)=j M I_{b} \sum_{p} \omega_{p} Z\left(\omega_{p}\right) \times e^{j k \Delta \phi}\langle\tau\rangle(t)
$$

$\langle\tau\rangle(t)=\hat{\tau} e^{j \Omega t}$ is the mean displacement of the bunches and can be understood either as the average over all particles of a same bunch or as the average over all bunches of the train; Impedance is sampled at frequencies $\omega_{p}=(p M+n) \omega_{0}+\Omega$.

When all particles have the same incoherent synchrotron frequency, we obtain the well-known growth rate of the $\mathrm{n}^{\text {th }}$ mode, after substitution of the induced voltage (2) into the single particle equation of motion (1):

$$
\frac{1}{\tau}=\frac{\alpha I_{0}}{4 \pi E / e Q_{s}} \sum_{p} \omega_{p} \Re e\left[Z\left(\omega_{p}\right)\right] e^{-\left(\omega_{p} \sigma_{t}\right)^{2}}-\frac{1}{\tau_{s}}
$$

In a similar way, we obtain the well-known result for the transverse multi-bunch instability:

$$
\frac{1}{\tau}=-\frac{\beta_{\perp} \omega_{0} I_{0}}{4 \pi E / e} \sum_{p} \Re e\left[Z_{\perp}\left(\omega_{p}\right)\right] e^{-\left(\omega_{p} \sigma_{t}\right)^{2}}-\frac{1}{\tau_{\perp}}
$$

with the longitudinal and transverse damping times $\tau_{s}$ and $\tau_{\perp}$, and the mode spectrum lines $\omega_{p}=(p M+n) \omega_{0}+\omega_{s}$ and $\omega_{p}=(p M+n) \omega_{0}+\omega_{\beta}$ for longitudinal dipole mode and rigid dipole transverse oscillations, respectively.

Independently of the vacuum chamber impedance, we 
can compare the sensitivity of different machines to longitudinal or transverse CBI by means of the parameters:

$$
S_{L C B I}=\frac{\alpha \tau_{s}}{2 E / e Q_{s}} \quad \text { and } \quad S_{T C B I}=\frac{\beta_{\perp} f_{0} \tau_{\perp}}{2 E / e}
$$

The sensitivity is of course inversely proportional to the beam energy and to the longitudinal or transverse focusing strength. Table 1 gives parameter values in the longitudinal plane for some colliders and synchrotron light sources.

Table 1: LCBI sensitivity parameter for some machines

\begin{tabular}{|lcccc|}
\hline & KEK-B & PEP-II & CESR III & DA $\Phi$ NE \\
$E(\mathrm{GeV})$ & 3.5 & 3.1 & 5.3 & 0.510 \\
$S_{L}$ & 3.7 & 22.7 & 41.4 & 3141 \\
\hline & ESRF & ELETTRA & ALS & SOLEIL \\
$E(\mathrm{GeV})$ & 6 & 2 & 1.9 & 2.5 \\
$S_{L}$ & 1 & 32.2 & 34.7 & 6.2 \\
\hline
\end{tabular}

The cures against CBI, developed at various places, are reviewed hereafter and systematically applied to the SOLEIL synchrotron light source for illustration.

\section{LANDAU DAMPING}

Landau damping proved to be an efficient tool in stabilizing multi-bunch instabilities, especially at ESRF, where this method is successfully used for the operation [5]. When the oscillators, which represent either the particles in a bunch or the different bunches in the train, have a finite spectrum of natural frequency with a distribution $\rho(\omega)$, the net response of the beam to the driving force due to wakefields in (1) is failing and the beam can be stable again if the frequency spread is large enough. The substitution of the induced voltage (2) by all oscillators with different frequencies into the single particle equation of motion (1) leads to the dispersion relation

$$
-\Delta \Omega_{0} \times 2 \omega_{s} \int \frac{\rho(\omega) d \omega}{\omega^{2}+2 j \alpha_{s} \Omega-\Omega^{2}}=1
$$

$$
\text { with } \quad \Delta \Omega_{0}=-\frac{\alpha I_{0}}{4 \pi E / e Q_{s}} \sum_{p} j \omega_{p} Z\left(\omega_{p}\right),
$$

the coherent frequency shift in the absence of Landau and synchrotron dampings. When frequency spread is provided by point-like bunches in the train, the integral should be replaced by a summation over the individual bunches, though the integral might be kept for many bunches.

\section{1 rf voltage modulation}

A first source of bunch frequency spread can come from the modulation of the $r f$ voltage, easily provided by beam loading in the rf cavity with partial filling of the ring. In the steady-state, the phase of the bunches grows about linearly. As a result, the frequency distribution consists in a nearly rectangular spectrum, with total spread and phase modulation

$$
\begin{aligned}
\frac{\Delta \omega}{\omega}=\frac{1}{2} \frac{\Delta V}{V} & =\tan \Delta \phi \cdot \tan \phi_{s}, \\
\Delta \phi & =\frac{\omega}{2 V} R / Q I_{0} \Delta T_{g a p}
\end{aligned}
$$

At ESRF, the instability threshold has been increased by this way, from about $60 \mathrm{~mA}$ to beyond the nominal intensity of $200 \mathrm{~mA}$ with a 1/3 filling.

A stability diagram, corresponding to the dispersion relation (5), is usually defined in the complex plane by plotting on the one hand the locus of the inverse of the integral as $\Omega$ is swept from $-\infty$ to $+\infty$, and the frequency shift $\Delta \Omega_{0}$ without Landau and radiation dampings on the other hand. Figure 2 shows for example the stability diagram of the SOLEIL ring, assuming LEP $\mathrm{Cu}$ cavities, operating at $352 \mathrm{MHz}$ and having a first $\mathrm{HOM}$ at about $500 \mathrm{MHz}\left(\mathrm{R} / \mathrm{Q}=75 \Omega\right.$ and $\left.\mathrm{Q}=3 \cdot 10^{4}\right)$. As the frequency of the cavity mode is not exactly known, it is also scanned and the frequency shift $\Delta \Omega_{0}$ just looks like the resonance curve of the HOM. Without Landau damping, the instability threshold is $14 \mathrm{~mA}$, assuming perfect overlap of the HOM with a spectral line of the beam. To achieve a threshold $I_{t h}$, a rectangular spectrum must have a total frequency spread $\Delta \omega$ given by

$$
\frac{\alpha I_{t h} \omega_{r} R_{s}}{4 \pi E / e Q_{s}}=\frac{\Delta \omega}{\pi / 2-\operatorname{ArcTan}\left(\alpha_{s} / \Delta \omega\right)}
$$

For $100 \mathrm{~mA}$, the required spread is larger than $6 \%$, whereas the frequency spread provided by the fractional filling is only $0.3 \%$ for a current of $100 \mathrm{~mA}$ and a $2 / 3$ filling of the SOLEIL ring, making this method impractical in this case.

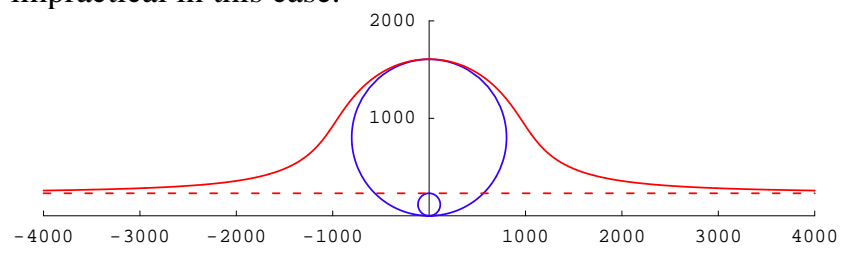

Figure 2: Stability diagram for the SOLEIL ring

- radiation damping only + HOM with $16 \mathrm{~mA}$

- rectangular spectrum $(\operatorname{spread}=6.3 \%)+$ HOM $(100 \mathrm{~mA})$.

Synchrotron frequency splitting can also be achieved by driving the normal RF cavities at a frequency $(h \pm 1) f_{0}$ and was used at CERN to suppress longitudinal instability [6].

In a similar way, aiming at producing a frequency spread even for an homogenous filling, an active $r f$ modulation has been tested in the ESRF storage ring by driving two of the four installed cavities at one revolution harmonic above the $r f$ frequency. The modulation frequency must be precisely $f_{0}$ to avoid longitudinal excitation of the beam. All the HOMs could be made in principle harmless well beyond the nominal intensity of $200 \mathrm{~mA}$. However, the $n=1$ instability prevents the cavities from being tuned close to the $h+l$ revolution 
harmonic. The trade-off between the modulation level and the reflected power due to detuning, results in a maximum current of $170 \mathrm{~mA}$.

\subsection{Landau cavity}

Since an harmonic cavity introduces non-linearities in the focusing force and thus some spread in synchrotron frequency, it has been proposed $[7,8,9]$ for stabilizing multi-bunch instabilities. The maximum frequency spread is achieved in bunchlengthening mode, when the slope of the total voltage is zero at the bunch location. With this quartic bucket potential, the distribution has the form [10]

$$
\rho(\omega)=K \omega^{2} e^{-\frac{1}{2}\left(\omega / \omega_{n}\right)^{4}}
$$

and has a maximum at $\omega=\omega_{n}$ which is generally much lower than the natural synchrotron frequency. A $3^{\text {rd }}$ harmonic cavity in the SOLEIL ring provides in this way a relative frequency spread of about $200 \%$, but since the center-frequency has been dramatically decreased, the net result is a poor improvement in the stabilization. The stability diagram with and without Landau cavity are shown in Fig.3. The gain on the instability threshold is only $30 \%$. We note that, operating in the bunchshortening mode, the same harmonic cavity would give a gain of about $\sqrt{2}$ on the threshold (the same as the synchrotron frequency enhancement) with a same voltage, about one third of the main $r f$ voltage.

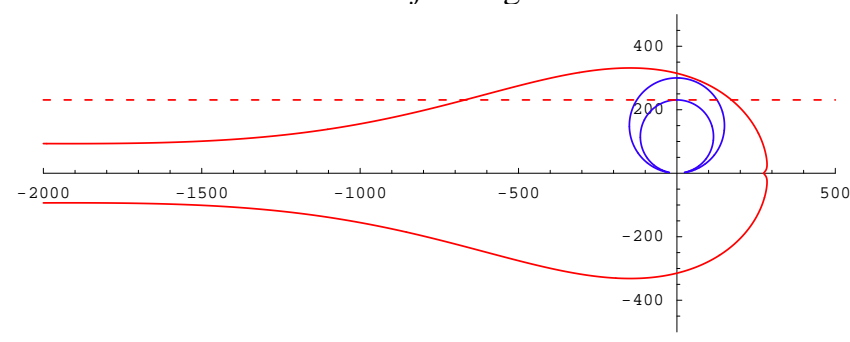

Figure 3: Stability diagram for the SOLEIL ring

- radiation damping only + HOM with $16 \mathrm{~mA}$

- spread from a 3rd harmonic cavity + HOM with 18 mA.

\subsection{Betatron tune spread}

Conversely to the longitudinal case, a significant spread in betatron frequency is easily obtained because of the nonlinearities in the focusing system or with a non-zero chromaticity, together with an energy spread in the beam. For that reason essentially, transverse multi-bunch instability occurs after longitudinal instability on most of the existing rings, whereas a crude threshold calculation gives generally the inverse. With Gaussian distribution in energy and betatron frequency spectrum $\sigma_{\omega}=Q_{\perp} \omega_{0} \xi \sigma_{E} / E$, the dispersion relation can be analytically calculated. Neglecting the transverse damping rate $\alpha_{\perp}$, the required spread is simply:

$$
\sigma_{\omega}>-\sqrt{\frac{\pi}{2}} \mathfrak{I} m\left(\Delta \Omega_{0}\right)
$$

For example, the transverse instability, excited by the most dangerous dipole HOM of the LEP $\mathrm{Cu}$ cavity $\left(\mathrm{f}_{\mathrm{r}}=614 \mathrm{MHz}, \mathrm{R}_{\perp} / \mathrm{Q}=360 \Omega / \mathrm{m}, \mathrm{Q}=6.10^{4}\right.$ ) has a current threshold of only $6 \mathrm{~mA}$. A chomaticity of only $\xi=0.1$ pushes away the onset of the instability to $240 \mathrm{~mA}$. The corresponding stability diagram is shown in Fig.4.

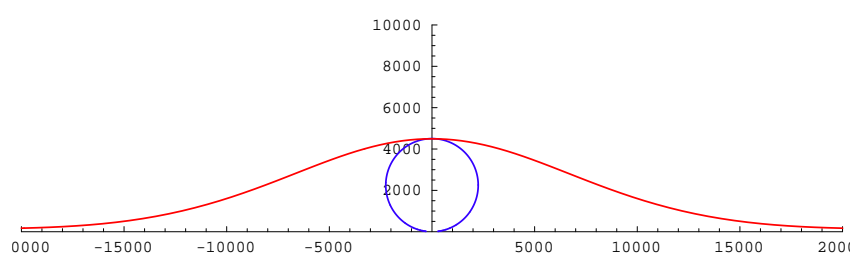

Figure 4: Stability diagram for the SOLEIL ring Gaussian betatron spread with chromaticity $\xi=0.1$ and energy spread $\sigma_{E} / E<10^{-3}$.

\section{MODE FREQUENCY CONTROL}

As the coupled-bunch modes are spaced one revolution frequency apart, there is some latitude in relatively small rings to escape the sharp HOMs from the beam spectrum lines, provided that they are not damped. This method was developed and is routinely used at ELETTRA [11], where the HOMs are tuned by precise cavity temperature control. Temperature windows, in which the growth rates of all modes are below the radiation damping rate, are first calculated. These stability windows can be checked and refined by a direct measurement of the coupled bunch mode spectrum on the machine. The growth rate of all longitudinal and transverse modes are calculated according to the expressions $(3,4)$ by summing the contribution of all cavities present in the machine. The resonance frequency $\omega_{k}$ of cavity mode $k$ is then a function of temperature $T$ and fundamental mode tuning $\omega_{f}$, being itself function of beam current; at first order :

$$
\omega_{k}\left(T, \omega_{f}\right)=\omega_{k}\left(T_{0}\right)+\frac{\partial \omega_{k}}{\partial T}\left(T-T_{0}\right)+\frac{\partial \omega_{k}}{\partial \omega_{f}}\left(\omega_{f}-\omega_{f 0}\right)
$$

Since it was difficult at ELETTRA to find temperature intervals, stable for both longitudinal and transverse planes, movable plungers were designed for allowing an additional degree of freedom [11]. Assuming six Elettratype cavities installed in the SOLEIL ring to provide 5 MV $r f$ voltage and $400 \mathrm{~kW} r f$ power to the beam, the cavity temperatures have been optimized by taking into account the nine longitudinal modes below the cut-off frequency of the beam tubes. The HOM spectrum has been randomly generated with a frequency spread of a few $\mathrm{MHz}$ for each cavity. Fig.5 shows for example one stability window of one cavity after optimization, for a temperature scan of $30^{\circ} \mathrm{C}$. Only the growth rates above the radiation damping rate are plotted. However, it was not always possible to find stability intervals for some HOM spectra ( $25 \%$ over 100 seeds) and movable plungers would be also required for that cases. 


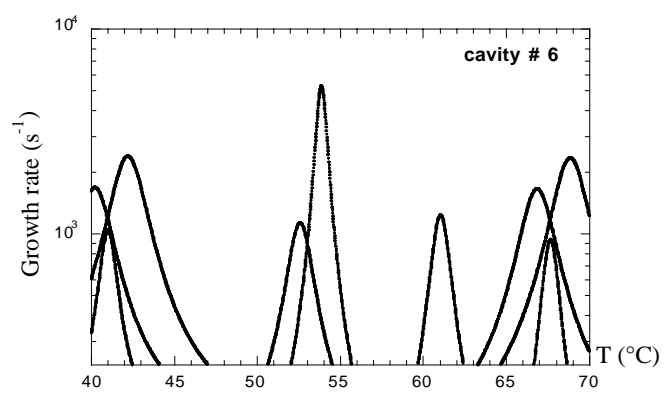

Figure 5: Example of stability window for one of the Elettra-type cavities in the SOLEIL ring.

\section{HEAVY MODE DAMPING}

The other way to combate CBI consists in grappling directly with the source of the instabilities, i.e. in damping as much as possible the HOMs in order to lower the resonant buildup of the fields. Once the deQing requirements are determined so that growth rates do not exceed the radiation or eventually feedback damping rates, dampers must be designed and their effectiveness checked with bench measurements. Various designs for superconducting and normal conducting cavities have been developed to meet high power and low impedance challenges. SC technology offers many advantages:

- Fewer cells, and thus lower overall impedance, for a given voltage, due to the high $\mathrm{CW}$ gradient capability

- Higher achievable deQing, because large beam holes are allowed, while keeping a very high accelerating mode shunt-impedance, through which HOMs can propagate out and be easily damped.

The drawbacks of SC systems are nevertheless a larger complexity, owing mainly to the cryogenic system, and the precautions that have to be taken against the risk of pollution of coupler and cavities, which could result in $\mathrm{Q}_{0}$ degradation or even quenches.

For NC cavities, dampers are mounted directly on the cavity walls at proper locations, where the coupling to the most troublesome modes is maximum. HOM power is carried out and dissipated on external $r f$ loads. Waveguide couplers, with a cut-off frequency slightly above the fundamental mode frequency, offer a natural FM rejection and provide higher deQing than coaxial couplers. Three ridged waveguides are generally placed symetrically around the nose-cone shaped cell to provide heavy damping $[12,13]$. Care must be paid to the additional power dissipation, due to field penetration into the waveguide, in the estimation of the thermal stress of the cavity wall at high field level.

Dampers cannot be directly mounted on the cavity walls of SC cavities (risk of multipactor, magnetic quench and surface contamination), but beam tubes can be made large enough for efficient coupling to the cavity modes.

Two approaches have been considered so far :

- Dampers are formed by the beam pipes themselves, by bonding $r f$ lossy material (ferrite) to the inner surface of both pipes, outside the cyostat (CESR, KEK-B)

- More classical HOM dampers are mounted on the beam pipes, in the vicinity of the cavity (LHC, SOLEIL)

The first option $[14,15]$ needs very large openings to ensure the propagation of all modes and the high HOM powers require that special attention be paid to the outgassing rate of the ferrite for preventing surface contamination. The second option allows the housing of several cells in a single cryostat, but puts more the issue on the HOM couplers, which must be optimized in combination with the string of cavities. A special arrangement, proposed for SOLEIL [16] and shown in Fig. 6 involves pairs of cavities, linked with large beam tubes in-between, but terminated on smaller outer pipes, featuring strong coupling for the HOMs and weak coupling for the accelerating mode.

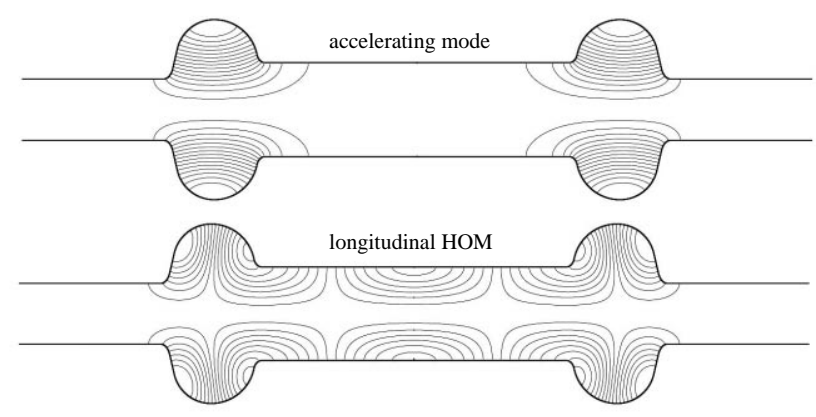

Figure 6: Cavity-pair arrangement for SOLEIL featuring weak coupling for the accelerating mode (top) and strong coupling for HOMs (bottom).

HOM dampers are mounted on the wide tubes, where the standing-wave HOM fields have large peaks. Inner tube diameter and cavity spacing have been numerically optimized and checked by bench measurements. Two coaxial couplers per cell, tailored to the deQing requirement of each $\mathrm{HOM}$, are enough to meet the SOLEIL specifications, with a safety margin of the order of 10 for both planes.

\section{FEEDBACK SYSTEMS}

Active feedback systems have been developed for more than 20 years [17], first in the frequency domain, on a mode-by-mode basis, and more recently in the time domain $[18,19,20]$, on a bunch-by-bunch basis, thanks to the advent of commercially available fast Digital Signal Processors. They are complementary to passive mode damping in the sense that they can damp definitely all coupled bunch modes, so long as impedances arising from strong HOMs have been first sufficiently reduced. Otherwise, and assuming that the coherent frequency shifts are smaller than the synchrotron frequency to prevent any coupling of internal modes, a huge broadband and very expensive power would be required at the kicker. The 
correction kick voltage needed to just cancel the beaminduced voltage causing growth of a coupled-bunch mode is

$$
\Delta V=I_{0} \Delta \phi \sum_{p} \frac{\omega_{p}}{\omega_{r f}} \Re e\left[Z\left(\omega_{p}\right)\right]
$$

where $\Delta \phi$ is the amplitude of phase modulation at the $r f$ frequency for the coupled-bunch mode.

Let's consider again with the first HOM of two LEP $\mathrm{Cu}$ cavities in the SOLEIL storage ring $\left(\mathrm{f}_{\mathrm{r}}=500 \mathrm{MHz}\right.$, $\mathrm{R} / \mathrm{Q}=75 \Omega$ and $\mathrm{Q}=3.10^{4}$ ), full coupling would call for a longitudinal kick of $84 \mathrm{kV} /$ turn, assuming a mode amplitude of $1.5^{\circ}$. The power required $P=\Delta V^{2} / 2 R_{s}$, would be more than $5 \mathrm{MW}$, assuming a DA $\Phi$ NE type kicker (low-Q resonator loaded with ridged waveguides, shunt impedance of about $700 \Omega$ ). In order to make the feedback damping rate practical, cavity modes must then be firstly reduced by nearly two orders of magnitude.

Any feedback system consists mainly of three parts:

- A detector for beam oscillations measurement

- A filter, analog or digital, for providing the proper time delay, corresponding to a $\pi / 2$ phase rotation, while suppressing the $d c$ component in the error signal

- A broadband amplifier and kicker for imparting the correction kick

The mode-by-mode feedback is generally used when only a few troublesome coupled-bunch modes have been identified. For a very large number of bunches, the bunchby-bunch approach is more attractive. In the latter case, the bunches are treated as individual oscillators and the minimum bandwidth of the feedback system must be, from the Nyquist theorem, half the bunch frequency to be able to damp all coupled-bunch modes. PEP-II, ALS and DAФNE have adopted a common longitudinal feedback system design, based on fast ADC/DAC converters and DSP chips for digital filtering. After digitizing of the baseband error signal, a N-taps FIR (Finite Impulse Response) filter, which performs a discrete-time convolution over $\mathrm{N}$ prior values of the error signal, is used for providing maximum gain at the synchrotron frequency and zero $d c$ response. Taking advantage of the relatively low synchrotron frequency, the number of processors needed is reduced by sampling at a rate slower than the revolution frequency. A detailed hardware description can be found in [19]. In addition, the DSP architecture revealed to be an efficient diagnostics tool, such as measurements of growth and damping rates by means of time domain transient techniques [21]. For KEK-B, the oscillation is damped simultaneously at both phase shifts $\pi / 2$ and $3 \pi / 2$, with gains of inverted signs, by using a simple and very fast (no multiplications) 2-tap digital filter [22]. Transverse feedback systems usually combine the signals of two PU, placed approximately $90^{\circ}$ in betatron phase apart, with appropriate coefficients to produce the $\pi / 2$ betatron phase shift for any kicker location and betatron tune. Signal processing (PU signals combining and time delaying) is preferably performed at baseband by a digital system [23].

\section{REFERENCES}

[1] A. Mosnier, "Cures of Coupled-Bunch Instabilities : Landau Damping, HOM Frequency Control, HOM Free Cavities and Feedbacks", SOLEIL Report 99-3, 1999.

[2] F.J. Sacherer, "A Longitudinal Stability Criterion for Bunches Beams", IEEE Trans. Nucl. Sci., NS-20, 825, 1973.

[3] J.L. Laclare, "Bunched Beam Coherent Instabilities", CERN 8703, Vol. I, pp. 264-326, 1987.

[4] A.W. Chao, "Physics of Collective Beam Instabilities in High Energy Accelerators", J. Wiley \& Sons, 1993.

[5] O. Naumann and J. Jacob, "Landau Damping of Longitudinal Instabilities for the Operation of the ESRF Storage Ring", Proc. of the 6th European Part. Acc. Conf., Stockholm, June 1998.

[6] D. Boussard and J. Gareyte, "Damping of the Longitudinal Instability in the CERN PS", Proc. of 8th Int. Conf. of High Energy Acc., CERN 1971.

[7] R. Bosch and C. Hsue, "Suppression of Longitudinal CoupledBunch Instabilities by a Passive Higher Harmonic Cavity", Proc. of Part. Acc. Conf., 1993, Vol. 42(2), pp. 81-99.

[8] S. Bartalucci et al, "A 3rd Harmonic Cavity for DAФNE", Proc. of the 4th European Part. Acc. Conf., London, June 1994.

[9] Å. Andersson et al, "Landau Cavities at MAX II", Proc. of the 6th European Part. Acc. Conf., Stockholm, June 1998.

[10] A. Hofmann, S. Myers, "Beam Dynamics in a Double RF System", Proc. of the 11th Int. Conf. on High-Energy Acc., Geneva, 1980.

[11] M. Svanderlik et al, "Improved Methods of Measuring and Curing Multibunch Instabilities in ELETTRA", Proc. of the 5th European Part. Acc. Conf., Sitges, June 1996.

[12] R. Boni, "High Power Test of the Waveguide Loaded RF cavity for the FrascatiI $\Phi$-Factory Main Rings", Proc. of the 5th European Part. Acc. Conf., Sitges, June 1996.

[13] R. Rimmer et al, "Higher Order Modes Damping Studies on the PEP-II B-Factory RF Cavity", Proc. of the 3rd European Part. Acc. Conf., Berlin, March 1992.

[14] T. Furuya et al, "Superconducting Accelerating Cavity for KEK B-Factory", Proc. of the 7th Workshop on RF Superconductivity, Gif-sur-Yvette, October 1995.

[15] S. Belomestnykh et al, "Development of Superconducting RF for CESR", Proc. of the 1997 Part. Acc. Conf., Vancouver.

[16] A. Mosnier et al, "Design of a Heavily Damped Superconducting Cavity for SOLEIL", Proc. of the 1997 Part. Acc. Conf., Vancouver.

[17] F. Pedersen and F. Sacherer, "Theory and Performance of the Longitudinal Active Damping System for the CERN PS Booster", IEEE NS-24, No 3, 1396 (1977).

[18] M. Bassetti et al, "DAФNE Longitudinal Feedback", Proc. of the 3th European Part. Acc. Conf., Berlin, June 1992.

[19] G. Oxoby et al, "Bunch-by-bunch Longitudinal Feedback System for PEP-II", Proc. of the 4th European Part. Acc. Conf., London, 1994.

[20] D. Teylerman et al, "Operation and Performance of the PEP-II Prototype Longitudinal Damping System at the ALS", Proc. of the 1995 Part. Acc. Conf., Dallas.

[21] S. Prabhakar, "Observation and Modal Analysis of CoupledBunch Longitudinal Instabilities via a Digital Feedback Control System", pub. in Particle Accelerators, 57/3, 1997.

[22] E. Kikutani, "Strategy for Developing Fast Bunch Feedback Systems for KEKB", Proc. of the 1997 Part. Acc. Conf., Vancouver.

[23] W. Barry et al, "Initial Commissioning Results from the PEP-II Transverse Coupled-Bunch Feedabck Systems", Proc. of the 6th European Part. Acc. Conf., Stockholm, June 1998. 